\title{
The Development of the Walking Assisting Device Using an Omnidirectional Mobile Robot
}

\author{
Tsubasa Sugimoto $^{\mathrm{a}}$, Yuhki Kitazono ${ }^{\mathrm{a}}$ \\ ${ }^{a}$ National Institute of Technology, Kitakyushu College, 5-20-1 Shii, Kokuraminami-ku, Kitakyushu-city Fukuoka 802- \\ 0985, Japan \\ *Corresponding Author: kitazono@kct.ac.jp
}

\begin{abstract}
Nowadays, many of the visually impaired people are living indoors or outdoors using a guide dog or a white cane. However, these walking assisting tools must often rely on the user's senses and free life of the visually impaired people are not yet guaranteed completely. For such reason, in recent years, the development of the walking assisting device with electronics have been actively carried out. But it is not popular yet in general. This study is the development of new walking assisting device for visually impaired people to live freely.
\end{abstract}

Keywords: visually impaired people, walking assisting device, ultrasonic sensor.

\section{Introduction}

Currently, serious aging society problem is in progress and people with some failure are increasing. According to the survey by the Ministry of Health, Labor and Welfare, the number of handicapped people in 2011 is 3.937 million people, it has been increasing year by year. ${ }^{(1)}$

Many visually impaired peoples go out with a guide dog or a white cane. In that case, users can go only in a location where they are familiar with. However, the range of their activities is restricted to their sense of direction, because it is impossible to get to a facility which is located at a place with which the user is unacquainted. If elderly people who had never keep a dog are suffering visual impairment, it is a great resistance for them to become the owner of a large dog. In addition, the training of a guide dog takes more than one year, all dogs trained not become a guide dog. Furthermore, it is very difficult to overtake the demand, because period is only eight years to work as a guide dog. In fact, the whole country in about 3,000 visually impaired person wants to guide dog. In contrast, the number of working dogs are about thousand. ${ }^{(2)}$ If they use a white cane, walking to the braille block or without braille block is difficult because they walk as they are depending on the braille block. Moreover, since they recognize the braille block as they are swinging left and right, the trouble is sometimes caused by the contact of the white cane, many case develop into some incidents. Elderly with a visual impairment cannot even possible to use a white cane, therefore they go out with their attendance or many visually impaired people do not all go out. In order to solve these problems, it is urgent need to robotize walking assisting tool.

To begin with, the roles of the white cane are (1. To ensure the safety of the user by the defense in front of the obstacles and danger), (2. To collect the information necessary to walk such as steps, sidewalk and braille block), (3. Reminder to healthy subjects who are in the same space). On the other hand, the role of guide dog is (1. To stop in front of the step of stairs), (2. To stop at an intersection), (3. To avoid obstacles, to support the walking), (4. To walk along the edge of the road).

The walking aid robots of current situation, there are those which guiding by recognizing the braille block by using the image processing, avoiding the obstacle by using a sensor such as an ultrasonic sensor and a laser range sensor and guiding by the use of a GPS and self-location estimation technology. ${ }^{(3-6)}$ These electronic walking assisting device is classified into two.

One is what is mechanized conventional guide dog or white cane, it is only to support to walk with the senses of the users mainly. The other lead the user by using such as GPS and image processing, and support for users to walk actively. Passive support, such as in the former case, users 
who can use is limited and their action range is restricted. Therefore, we will from now on it is required to develop active walking aid robot, such as in the latter case.

However, because a number of active walking support robots that have been developed up to now is a large size, there is a problem that it is difficult for users to go out with ease. Since the 1960s, even though the research of a walking assisting device has been actively carried out, because of the problem they haven't become common up to now. ${ }^{(7)}$

In 2011, in cooperative with Akita Seiko Co., Ltd. and Akita Prefectural University, the electronic white cane is developed and commercialized. This is what that detects obstacles in the ultrasonic sensor and inform the user by vibration. This product is a $270 \mathrm{~g}$ and lightweight, because a conventional white cane is almost the same shape, users can go out with ease. However, this is merely somewhat to solve the problem of wielding white cane, cannot exceed the range of the walking support functions of white cane. In this study, we propose a walking assisting device which can go out easily while performing active walking assisting and equipped with a large walking support device and the middle of the function of the electronic white cane.

\section{The structure and function}

\subsection{Structure}

The structure diagram of the walking assisting device is shown in Fig. 1. When users enter the direction they want to proceed, omni wheel robot which is a driving part is to induce them.

3WD48mm omni wheel robot platform by "Nexus robot" is used as driving part of this device. This is a platform set of omni-directional movable robot using a three-

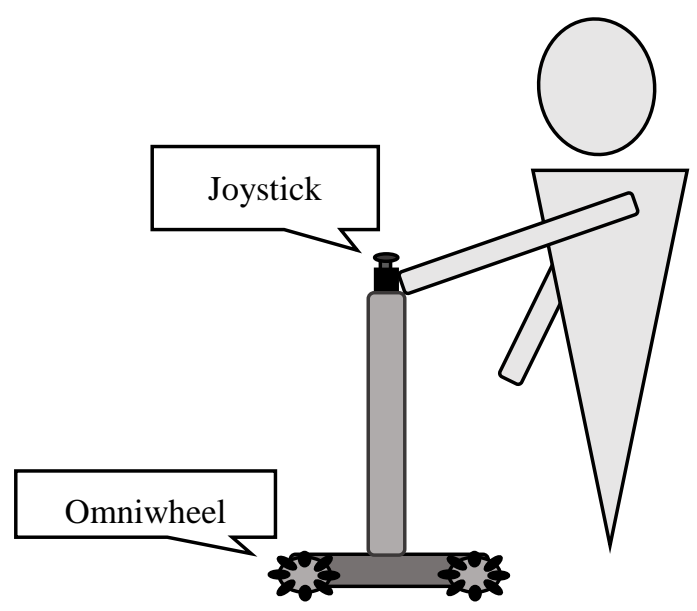

Fig. 1. The structure diagram of the walking assisting device

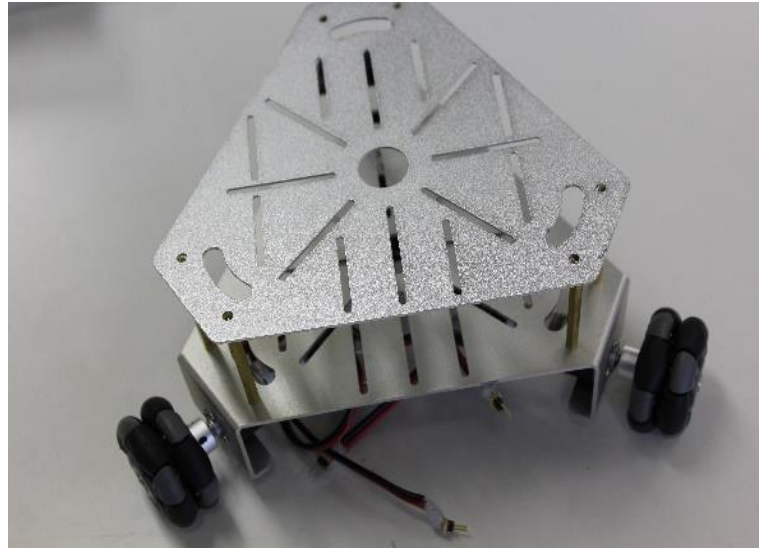

Fig. 2. 3-wheel overview of Omni wheel robot

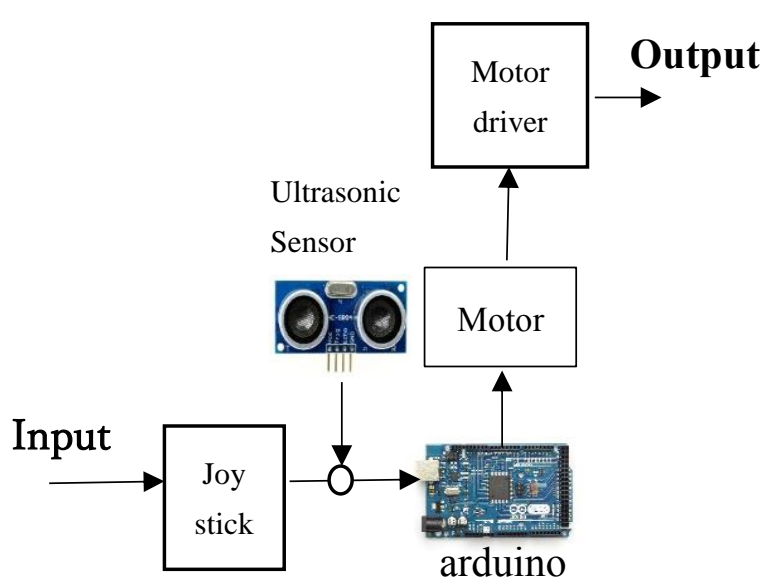

Fig. 3. Schematic view of the structure

wheeled omni wheel. The omni wheel, it is a unique wheel can be generated a driving force only in one direction by positioning a plurality of free rollers on the outer circumference of the wheel. A driving part of the omnidirectional movement mechanism was placed a minimum of three omni wheels every 120 degrees as the number of modules. The three-wheeled omni wheel is adopted as a drive unit, because it is lightweight and possible omnidirectional movement and can control easily. It shows the appearance in Fig. 2.

2-axis joystick that visually impaired people can input the direction and speed intuitively has been adopted as the input controller. Arduino is used as a control for the microcomputer. When users input directions that they want to proceed with the joystick, the device goes to these directions. Because the sensor to avoid obstacles is attached on this device, they may be able to walk in accordance with 


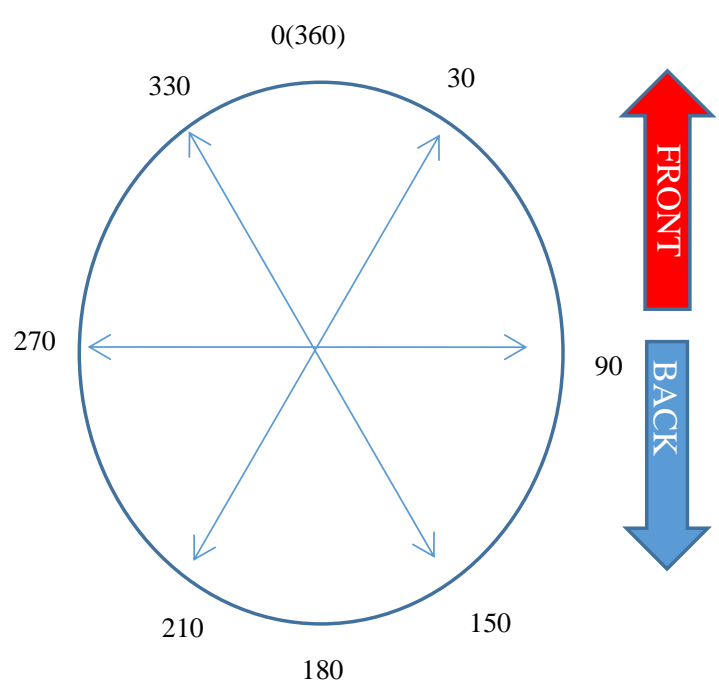

Fig. 4. Relationship diagram of the angle and the direction of travel

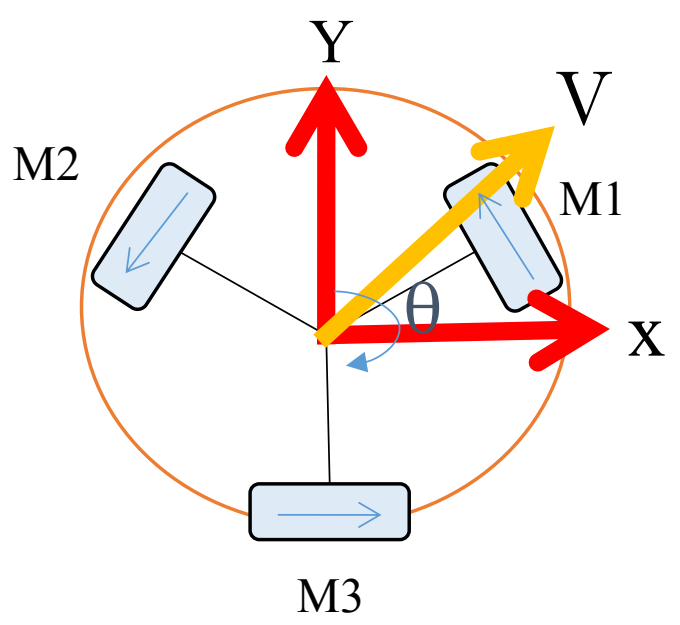

Fig. 5. Vector of the wheels and the traveling direction

this equipment. Sensors for detecting obstacles are ultrasonic sensor. Fig. 3. shows a schematic view of the structure.

\subsection{Input Analysis}

Analysis of the input data from the joystick is performed as follows. We read the angle and magnitude of the input data. Then, we set advancing to 0 degrees, the right 90 degrees, reverse 180 degrees, the left 270 degrees. Relationship angle and the traveling direction is shown in Fig. 4.

\subsection{Determination of the motor output}

Since omni wheels are set as Fig. 5., outputs of motors are determined to proceed a device in the direction of the vector $\mathrm{V}$. We define output ratio of each motors as M1, M2, $\mathrm{M} 3$, and the input as $\theta$, formulas for the output are
[Ratio]

\section{Output ratio of each motor}

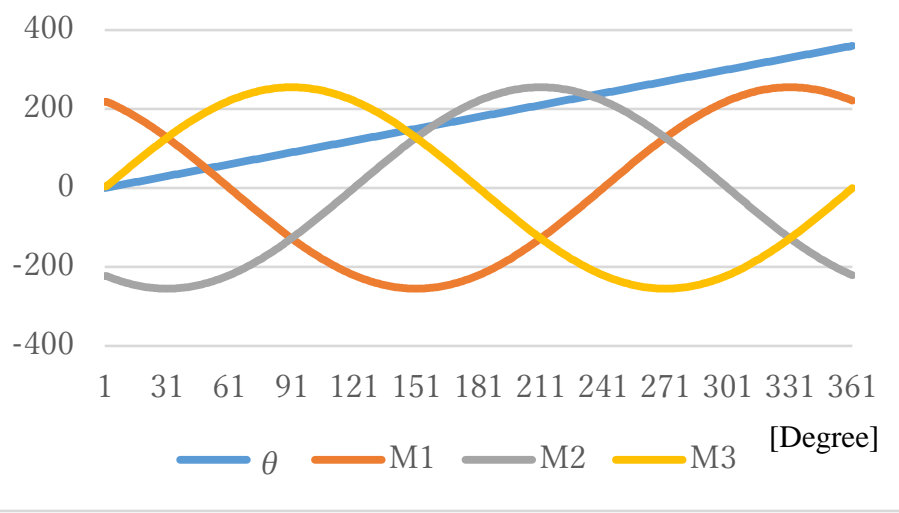

Fig. 6. Output ratio of each motor

determined as follows.

$$
\begin{aligned}
& M 1=-\sin \theta \\
& M 2=0.5 \sin \theta-\frac{\sqrt{3}}{2} \cos \theta \\
& M 3=0.5 \sin \theta+\frac{\sqrt{3}}{2} \cos \theta
\end{aligned}
$$

Positive values clockwise, a negative value is anti-clockwise. The output torque ratio of each motors when proceeding in the direction of each angle is determined. Fig. 6. is graph which show the output value of the motor when move to each direction. Moreover, it attaches the ultrasonic sensor in three directions, when the distance to the obstacle becomes less than $100 \mathrm{~mm}$, brakes the motor, and so as to ensure safety.

\subsection{Dimension and Weight}

The length of the cane to be attached to the threewheeled robot is material order to reduce weight in the $1 \mathrm{~m}$ is to use a vinyl chloride resin. Attached to a three-wheeled robot, finally, the height become $1.1 \mathrm{~m}$. Weight is $1294 \mathrm{~g}$. This is a much lighter than conventional guide dog robot. Then, one on the most important goal, that users can go out easily, is possible. Although the conventional guide dog robots cannot climb of stairs, because of this light, this device can go up and down stairs along the railing by lifting. In addition, because it is small compared to the guide dog robots, this can run also in the room of the narrow passage. 

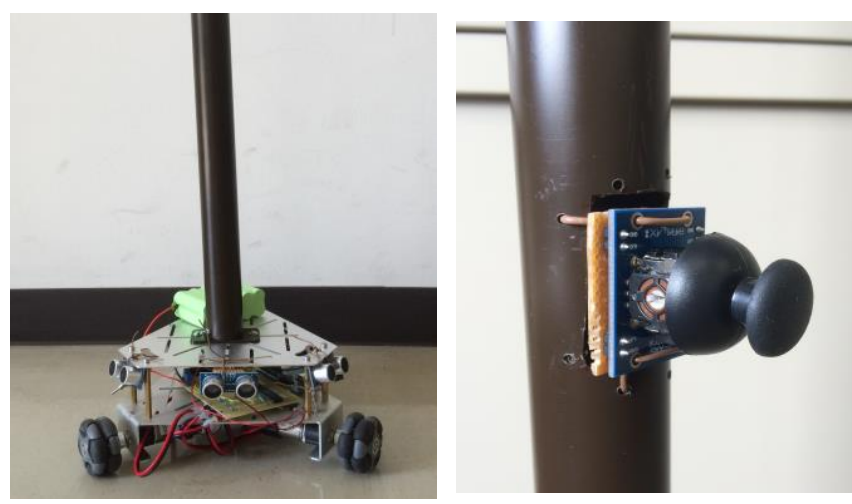

Fig. 7. The appearance of the walking assisting device

\subsection{Human interface}

In order to visually impaired people to recognize the direction of travel of the robot, attached the stick vertically. Thereby they can know the direction of travel in the tilt of the stick. The joystick is embedded to the stick as the input section, users can control with a cane naturally. Its appearance is shown in Fig. 7.

\section{Experiment}

Visual shielding using the eye mask, I confirmed the use of this device by walking the room. Even if we are visual shielding, we can be entered in the joystick sensuously, we can proceed freely to the direction wanting to proceed. If an obstacle is detected, this device can stop as specified, it did not strike or stumble. Because it is a lightweight, it could not climb any of the steps. However, we confirmed that we confirmed it to climb by lifting it. Thus we can use it like a white cane, when it climbs a step. If it is this shape, it is possible to perform actively walking support at light equipment.

\section{Conclusions}

In this study, we proposed a new form of the walking support machine which attaching a cane in all-directionsrobot. In addition, we consider that the shape of this device is applicable such as the elderly walking training as well as the visually impaired people. In this study, we can only develop functions like electronic white cane. However, in the future, it is scheduled to perform a walking aid made use of this shape by the use of a GPS. Further, we implement the function which not only stops by detecting obstacles by using a sensor, also avoids it and induce a safe passage.

\section{References}

(1) Cabinet Office, Government of Japan, “Annual Report on Government Measures for Peoples with disabilities (Summary) 2007"

(2) National Committee of Welfare for the Blind in Japan Self-support facility section guide dog committee: "Heisei 27 Annual report of guide dog training facility"

(3) Kotani Shinji, Kiyohiro Tomoaki, Mori Hideo: "Development of the Robotic Travel Aid for the Visually Impaired”, ITE Transactions on Media Technology and Applications Vol.51, No.6 pp.878 885, 1997

(4) Takeshi Kurata, Masakatsu Kourogi, Tomoya Ishikawa, Yoshinari Kameda, Kyota Aoki, Jun Ishikawa: "Navigation System for Visually-Impaired Pedestrians Preliminary Evaluation of Position Measurement and Obstacle Detection --“, IEICE Technical Report, Vol. 110, No. 238, pp. 67-72, 2010

(5) API Co. Ltd.: "Smart Electronics White Cane System", http://www.api-kk.com/denshi-hakujo/

(6) Kazuo Kawada, Toru Yamamoto, Yasuhiro Mada, Takuya Tsutsui: "Development of an Intelligent White Cane for Visually Handicapped Persons", The JSME Symposium on Welfare Engineering, Vol. 2002, No. 2-34, pp. 241-244, 2002

(7) Yoshikazu Seki: "Transition of Assistive Technologies for Mobility of Blind People", IEICE Technical Report, Vol. 111, No.58, pp87-90, 2011 